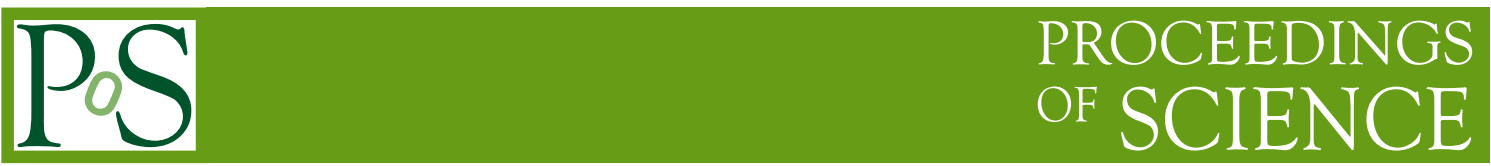

\title{
Coulomb phase stability and quark confinement
}

\author{
Manuel Asorey* and Alessandro Santagata \\ Departamento de Física Teórica. Facultad de Ciencias \\ Universidad de Zaragoza. 50005 Zaragoza. Spain \\ E-mail: asorey@unizar.es,alessandro.santagata@unizar.es
}

\begin{abstract}
Quantum Chromodynamics smoothly interpolates from an asymptotic freedom regime to a confinement regime. The transition is tracked by the instability of Coulomb phase in heavy quark backgrounds for intermediate values of the coupling constant. The quark-antiquark Coulomb phase holding at short distances leads at large distances to another phase driven by thick stringlike configurations. The critical distance where the transition occurs has a non-pertubative dependence on the coupling constant. The phenomenon can be derived in a straightforward way from first principles which opens a window to a new analytic approach to quark confinement.
\end{abstract}

From quarks and gluons to hadronic matter: A bridge too far?

2-6 September, 2013

European Centre for Theoretical Studies in Nuclear Physics and Related Areas (ECT*), Villazzano, Trento (Italy)

${ }^{*}$ Speaker. 


\section{Introduction}

The analytic approach to QCD from first principles has been very successful in the two extreme coupling constant regimes. At weak coupling $(\alpha \ll 1)$ perturbation theory shows that the $\beta$ function is negative which points out the asymptotic freedom behaviour of the theory at short distances. On the other extreme strong coupling expansion also shows that for large values of the coupling constant $(\alpha \gg 1)$ the theory is confining. However, to show that both behaviours arise in the same theory one needs to prove that there is no phase transition at intermediate coupling constant regimes. Otherwise, we will have two different theories with different ultraviolet and infrared behaviours. The absence of critical phenomena for non-vanishing values of the coupling constant has only been shown by numerical simulations on the lattice. The analytic proof of this fact has remained elusive for decades and still is one of the challenging theoretical questions in QCD. The only analytic tools which have explored the intermediate regime of coupling constants are large $N$ expansions and gauge-gravity dualities raised by AdS/CFT correspondence. However, the behaviour of real QCD at the intermediate regime of coupling constant still remains as an open problem from an analytic perspective. On the other hand the intermediate regime is very interesting from a phenomenological viewpoint. The physics of meson and baryon resonances provides a rich family of phenomena which require the precise understanding of the behaviour of QCD at intermediate energies.

Recently, a new approach due to Gribov [1][2] has emerged with new insights coming from QCD instability in heavy quarks backgrounds for large values of the effective $\alpha$ coupling constant. The instability would imply a vacuum decay on light quark-antiquark pairs [3][4]. A further analysis based on Dyson-Schwinger equations for the light quark Green functions lead to the existence of a critical value of the strong coupling $\alpha_{G}=\frac{3 \pi}{4}(1-\sqrt{2 / 3})$ beyond which the theory becomes unstable [3]-[6]. The analysis of the stability of Euclidean functional integral in one heavy quark background points out the existence of similar unstabilities for values of the coupling constant larger than critical value $\alpha_{c}=\sqrt{2}$ [7][8]. A similar critical value was found in earlier analyses [9]-[20] of Yang-Mills equations in a Coulomb background. However, one quark backgrounds do not respect the chromodynamics Gauss law and are unphysical. A more realistic setup is a quarkantiquark background which can correspond to a meson background matching chromodynamics Gauss law and all other physical constraints. In this note we analyse the stability properties of the Coulomb regime in this background finding some surprising features.

\section{Coulomb phase in a heavy quark-antiquark background}

Let us consider a pair of heavy static quark-antiquark. The Euclidean functional integral defined the Euclidean action

$$
S^{Y M}(A)=-\frac{1}{2 g^{2}} \int d^{4} x \operatorname{tr} F^{\mu v} F_{\mu v}-2 i \int d x^{0} \operatorname{tr}^{3}\left[A_{0}\left(\vec{x}+L \overrightarrow{\mathrm{e}}_{3}, x_{0}\right)-A_{0}\left(\vec{x}-L \overrightarrow{\mathrm{e}}_{3}, x_{0}\right)\right],
$$

is dominated by the imaginary Coulomb background solution of Euclidean Yang-Mills equations

$$
A_{0}(\vec{x})=\frac{i \alpha \mathrm{T}^{3}}{\left|\vec{x}-L \overrightarrow{\mathrm{e}}_{3}\right|}-\frac{i \alpha \mathrm{T}^{3}}{\left|\vec{x}+L \overrightarrow{\mathrm{e}}_{3}\right|}, \quad \vec{A}=0
$$


where $\alpha=\frac{g^{2}}{4 \pi}, 2 L$ is the distance between the two quarks and $\mathrm{T}^{3}$ is the third Gell-Mann matrix $\left(\mathrm{T}_{3}^{2}=-1 / 2\right)$. Instabilities can arise in second order fluctuations from non-abelian static magnetic field perturbations of the form

$$
\vec{\tau}(x)=\frac{\vec{x} \times \overrightarrow{\mathrm{e}}_{3}}{\rho^{\frac{3}{2}}} \phi(\rho, z) \mathrm{T}_{12}, \quad \tau_{0}=0,
$$

where $\mathrm{T}_{12}$ is any normalized linear combination of the first two components of Gell-Mann matrices $\left(\mathrm{T}_{12}^{2}=-1 / 2\right)$ and $\phi(\rho, z)$ satisfies the eigenvalue equation

$$
\left[\frac{\partial^{2}}{\partial \rho^{2}}+\frac{\partial^{2}}{\partial z^{2}}-\frac{3}{4 \rho^{2}}+\left(\frac{\alpha}{\sqrt{\rho^{2}+(z-L)^{2}}}-\frac{\alpha}{\sqrt{\rho^{2}+(z+L)^{2}}}\right)^{2}\right] \phi(\rho, z)=\lambda^{2} \phi(\rho, z)
$$

in cylindric coordinates.

The singularities associated to point-like structure of the heavy quarks need to be renormalized. This can be achieved in terms of boundary conditions which guarantee the selfadjointess of the second order differential operator involved in second order fluctuations (2.4).

The boundary conditions can be given in terms of zero modes around the singularities of this operator (2.4) which are given by

$$
\psi_{>}^{ \pm}(\rho, z)=\rho^{\frac{3}{2}}\left(\rho^{2}+(z-L)^{2}\right)^{-\frac{3}{4} \pm \frac{v}{2}}
$$

and

$$
\psi_{<}^{ \pm}(\rho, z)=\rho^{\frac{3}{2}}\left(\rho^{2}+(z+L)^{2}\right)^{-\frac{3}{4} \pm \frac{v}{2}}
$$

where $v=\sqrt{\frac{9}{4}-\alpha^{2}}$. Both solutions are normalizable for $\alpha^{2}>\frac{5}{4}$ and $v$ becomes imaginary for $\alpha^{2}>\frac{9}{4}$. Thus, for $\alpha^{2}<\frac{5}{4}$ the operator is essentially selfadjoint and has a unique selfadjoint extension with positive spectrum. In that regime the Coulomb phase is stable. For $\alpha^{2}>\frac{5}{4}$ general solutions can depends on a dimensionfull parameter $\Lambda$

$$
\begin{aligned}
& \psi_{>}(\rho, z)=\left(\frac{\rho}{\sqrt{\rho^{2}+(z-L)^{2}}}\right)^{\frac{3}{2}}\left[\left(\Lambda \sqrt{\rho^{2}+(z-L)^{2}}\right)^{v}-\left(\Lambda \sqrt{\rho^{2}+(z-L)^{2}}\right)^{-v}\right] \\
& \psi_{<}(\rho, z)=\left(\frac{\rho}{\sqrt{\rho^{2}+(z+L)^{2}}}\right)^{\frac{3}{2}}\left[\left(\Lambda \sqrt{\rho^{2}+(z+L)^{2}}\right)^{v}-\left(\Lambda \sqrt{\rho^{2}+(z+L)^{2}}\right)^{-v}\right]
\end{aligned}
$$

which introduces an anomalous breaking of conformal symmetry.

In such a case there is a family of boundary conditions for the operator involved in second order fluctuations $\phi$ parametrized by $\Lambda$, which make (2.4) selfadjoint and are given by

$$
\lim _{z \rightarrow L}\left(\phi^{\prime} \psi_{<}-\phi \psi_{<}^{\prime}\right)=0, \quad \lim _{z \rightarrow-L}\left(\phi^{\prime} \psi_{>}-\phi \psi_{>}^{\prime}\right)=0,
$$

where $\phi^{\prime}$ and $\psi^{\prime}$ denote the normal derivatives. 
However, there is a difference between the the strong coupling case $\alpha^{2}>\frac{9}{4}$ and the intermediate coupling case $\frac{5}{4}<\alpha^{2}<\frac{9}{4}$. In fact, the situation is reminiscent of the a single quark background where in the strong coupling regime there is an infinity of negative modes which lead to a strong instability of Coulomb phase, whereas in the intermediate regime only one negative mode points out a mild instability of that phase [7,8].

\section{Coulomb phase in the intermediate coupling constant regime}

Unlike in the one-quark background setup the spectral problem of the quark-antiquark background cannot be solved analytically. In a neighbourghood of each quark $\rho \simeq 0$ and $z \simeq \pm L$ a good approximation to the spectral problem for negative eigenvalues $-\lambda^{2}$ reduces to

$$
\left[\frac{\partial^{2}}{\partial \rho^{2}}+\frac{\partial^{2}}{\partial w^{2}}-\frac{3}{4 \rho^{2}}+\left(\frac{\alpha}{\sqrt{\rho^{2}+w^{2}}}-\frac{\alpha}{2 L}\right)^{2}\right] \phi(\rho, w)=\lambda^{2} \phi(\rho, w)
$$

where $w=z \mp L$. In the intermediate regime of coupling constants $\frac{5}{4}<\alpha^{2}<\frac{9}{4}$, for $\alpha<2 L \lambda$ there is only one real solution matching the boundary conditions. It can be given in terms of Whittaker function

$$
\phi(\rho, w)=\frac{\rho^{3 / 2}}{\rho^{2}+w^{2}} W\left(-\frac{\alpha^{2}}{\sqrt{4 L^{2} \lambda^{2}-\alpha^{2}}}, v, \frac{\sqrt{4 L^{2} \lambda^{2}-\alpha^{2}}}{L} \sqrt{\rho^{2}+w^{2}}\right),
$$

From the asymptotic expansion of Whittaker function near the origin $\rho \simeq 0, w \simeq 0$ it is easy to show that boundary conditions reduce to

$$
\frac{\Gamma[1-2 v]}{\Gamma\left[\frac{1}{2}-v+\frac{\alpha^{2}}{\sqrt{4 L^{2} \lambda^{2}-\alpha^{2}}}\right]}-\frac{\Gamma[1+2 v]}{\Gamma\left[\frac{1}{2}+v+\frac{\alpha^{2}}{\sqrt{4 L^{2} \lambda^{2}-\alpha^{2}}}\right]}\left(\frac{L \Lambda}{\sqrt{4 L^{2} \lambda^{2}-\alpha^{2}}}\right)^{2 v}=0
$$

Notice that there is a singularity at $v=\frac{1}{2}$, i.e. $\alpha^{2}=2$.

We shall restrict ourselves to the special intermediate regime $2<\alpha^{2}<\frac{9}{4}$. For the rest of the intermediate regime $\frac{5}{4}<\alpha^{2}<2$ the boundary conditions $\psi_{>}^{+}, \psi_{<}^{+}$in (2.5), (2.6) preserve conformal invariance and do not displays unstable modes.

The negative eigenvalue $-\lambda^{2}$ obtained from that condition increases as the distance $2 L$ between the two quarks decreases suggesting a possible vanishing for a finite critical distance $L=L_{c}$. The exact solution is very close to the approximate solution (3.3) (see Figure 1).

To find out the critical distance $L_{c}$ where the unstable modes disappear we have to go beyond $\alpha=2 L \lambda$. For $\alpha>2 L \lambda$ the solution (3.2) becomes complex and we get two independent real solutions matching the physical boundary conditions. This is in agreement with the exact solution of the negative eigenvalue problem, where there are two different solutions: $\phi_{s}$ which is parity symmetric and $\phi_{a}$ which is antisymmetric. The corresponding eigenvalues are slightly different, being the lowest the symmetric mode which is leading the instability. The approximate real solution corresponding to the exact symmetric solution is

$$
\phi_{s}(\rho, w) \simeq \frac{\rho^{3 / 2}}{\rho^{2}+w^{2}} \operatorname{Im}\left[W\left(-\frac{\alpha^{2}}{\sqrt{4 L^{2} \lambda^{2}-\alpha^{2}}}, v, \frac{\sqrt{4 L^{2} \lambda^{2}-\alpha^{2}}}{L} \sqrt{\rho^{2}+w^{2}}\right)\right]
$$




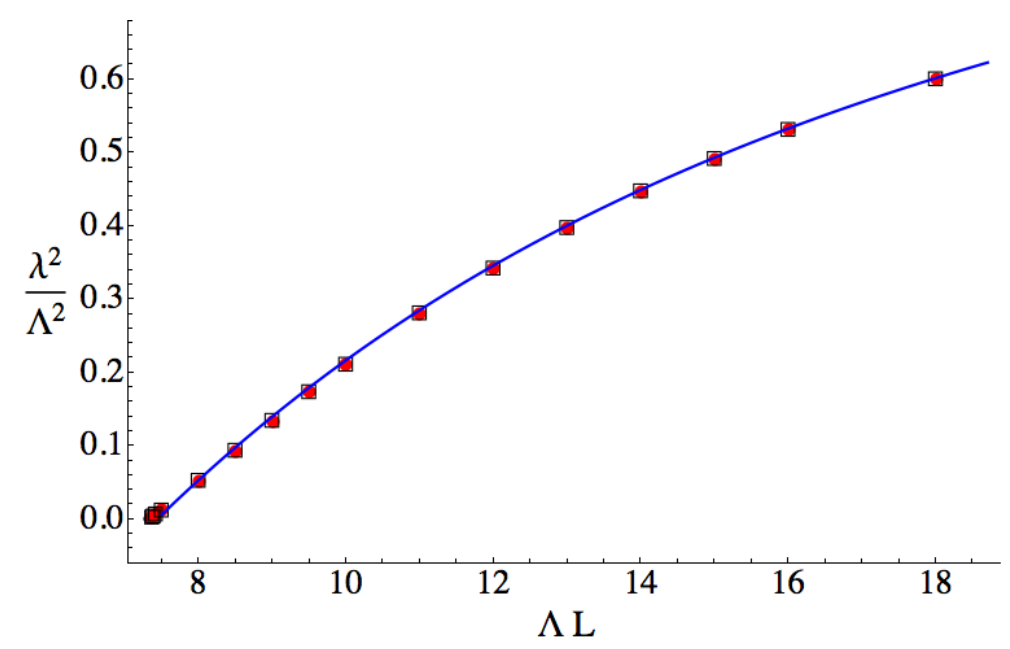

Figure 1: Behaviour of negative unstable modes of gluon fluctuations with the size $2 L$ of a heavy quark-antiquark pair at $\alpha=1.495$ in the special intermediate coupling regime. Red dots are exact values for the symmetric mode whereas the squares are the eigenvalues of the antisymmetric unstable modes. The blue curve represents the approximate values obtained by the analytic formula (3.1). Both results point out the existence of a critical size $2 L_{c}$ in this intermediate coupling regime where the unstable modes become stable.

with the boundary condition

$$
\operatorname{Im}\left[\frac{\Gamma[1-2 v]}{\Gamma\left[\frac{1}{2}-v+\frac{\alpha^{2}}{\sqrt{4 L^{2} \lambda^{2}-\alpha^{2}}}\right]}-\frac{\Gamma[1+2 v]}{\Gamma\left[\frac{1}{2}+v+\frac{\alpha^{2}}{\sqrt{4 L^{2} \lambda^{2}-\alpha^{2}}}\right]}\left(\frac{L \Lambda}{\sqrt{4 L^{2} \lambda^{2}-\alpha^{2}}}\right)^{2 v}\right]=0
$$

and the other one corresponding to the antisymmetric solutions is

$$
\phi_{a}(\rho, w) \simeq \frac{\rho^{3 / 2}}{\rho^{2}+w^{2}} \operatorname{Re}\left[W\left(-\frac{\alpha^{2}}{\sqrt{4 L^{2} \lambda^{2}-\alpha^{2}}}, v, \frac{\sqrt{4 L^{2} \lambda^{2}-\alpha^{2}}}{L} \sqrt{\rho^{2}+w^{2}}\right)\right]
$$

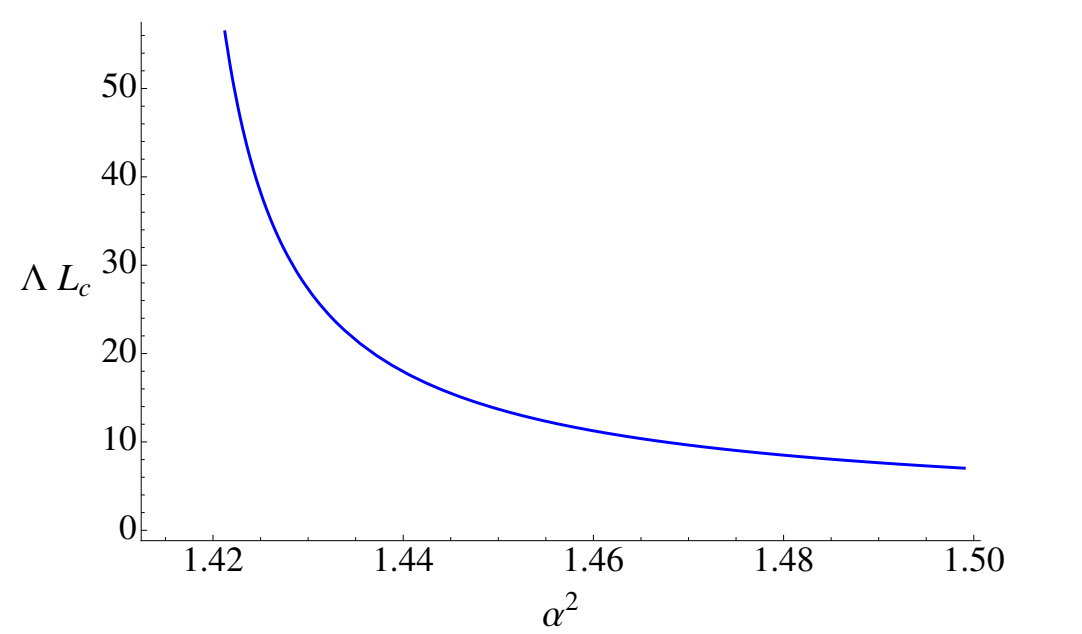

Figure 2: $\alpha$-dependence in the intermediate regime of the critical heavy meson size $2 L_{c}$ where negative unstable modes of gluon fluctuations become stable. The symmetric and antisymmetric modes are so overlapped that their difference cannot be appreciated in the plot, but the symmetric mode is always lower that the antisymmetric one 


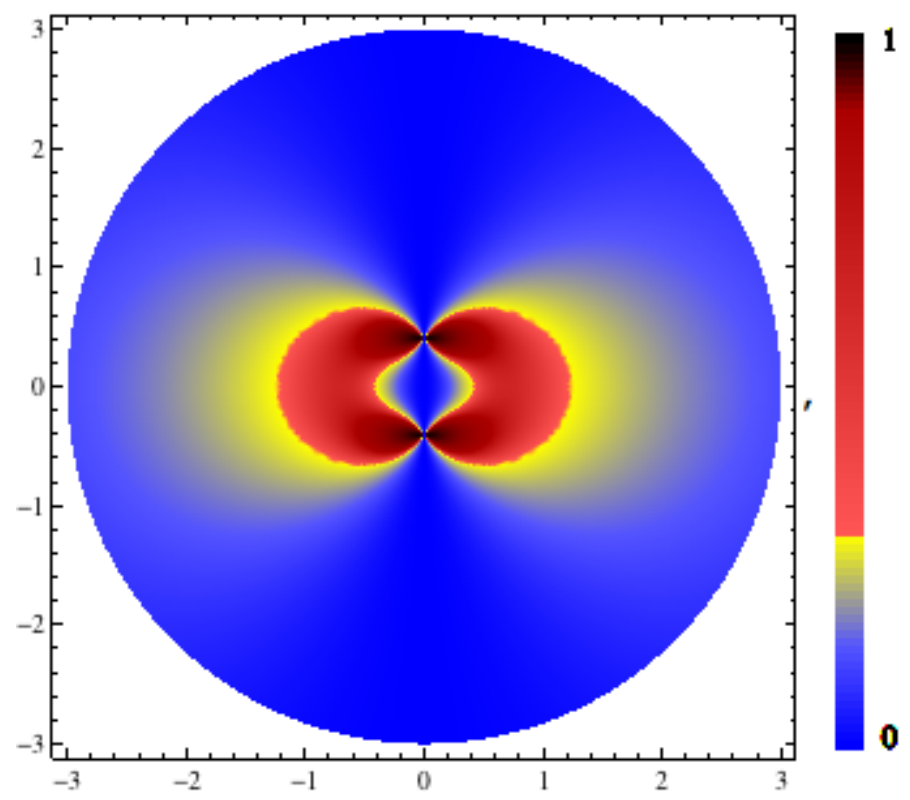

Figure 3: Density plot of the symmetric unstable mode of gluon fluctuations in a heavy quark-antiquark background. The configuration has a shape of a thick string connecting the two quarks, which is pointing to a confinement background.

with the boundary condition

$$
\operatorname{Re}\left[\frac{\Gamma[1-2 v]}{\Gamma\left[\frac{1}{2}-v+\frac{\alpha^{2}}{\sqrt{4 L^{2} \lambda^{2}-\alpha^{2}}}\right]}-\frac{\Gamma[1+2 v]}{\Gamma\left[\frac{1}{2}+v+\frac{\alpha^{2}}{\sqrt{4 L^{2} \lambda^{2}-\alpha^{2}}}\right]}\left(\frac{L \Lambda}{\sqrt{4 L^{2} \lambda^{2}-\alpha^{2}}}\right)^{2 v}\right]=0
$$

were we have used asymptotic expansion for the Whittaker near the origin.

There are also two critical distances which correspond to the distances where the symmetric and antisymmetric modes become stable. They can be obtained also from the approximate symmetric solution

$$
\phi_{s}(\rho, w)=\frac{\rho^{3 / 2}}{\rho^{2}+w^{2}} \operatorname{Im}\left[W\left(i \alpha, v, \frac{i \alpha}{L} \sqrt{\rho^{2}+w^{2}}\right)\right]
$$

and the approximate antisymmetric solution

$$
\phi_{a}(\rho, w)=\frac{\rho^{3 / 2}}{\rho^{2}+w^{2}} \operatorname{Re}\left[W\left(i \alpha, v, i \frac{\alpha}{L} \sqrt{\rho^{2}+w^{2}}\right)\right],
$$

respectively. The corresponding critical distances are

$$
\widetilde{L}_{c}=\frac{\alpha}{\Lambda}\left(\frac{\Gamma[1-2 v]}{\Gamma[1+2 v]} \frac{\operatorname{Im}\left[\frac{i^{\frac{1}{2}+v}}{\Gamma\left[\frac{1}{2}-i \alpha-v\right]}\right]}{\operatorname{Im}\left[\frac{i^{\frac{1}{2}}(-i)^{v}}{\Gamma\left[\frac{1}{2}-i \alpha+v\right]}\right]}\right)^{\frac{1}{2 v}}
$$

and

$$
\widetilde{L}_{c}^{\prime}=\frac{\alpha}{\Lambda}\left(\frac{\Gamma[1-2 v]}{\Gamma[1+2 v]} \frac{\operatorname{Re}\left[\frac{i^{\frac{1}{2}+v}}{\Gamma\left[\frac{1}{2}-i \alpha-v\right]}\right]}{\operatorname{Re}\left[\frac{i^{\frac{1}{2}}(-i)^{v}}{\Gamma\left[\frac{1}{2}-i \alpha+v\right]}\right]}\right)^{\frac{1}{2 v}}
$$




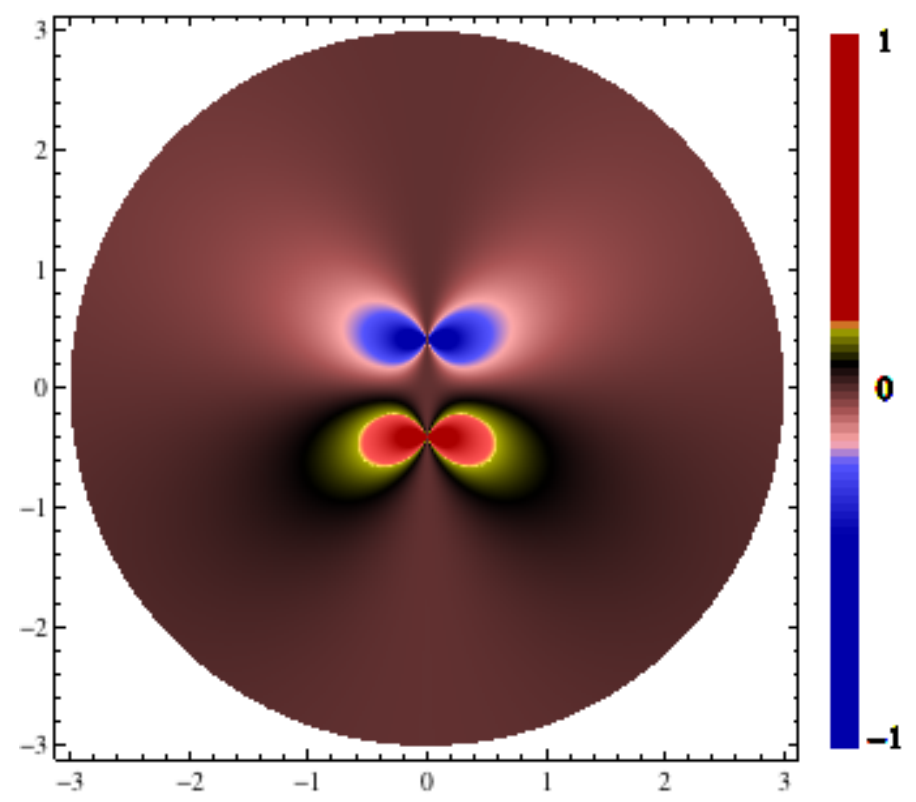

Figure 4: Density plot of the antisymmetric unstable mode of gluon fluctuations in a heavy quark-antiquark background. The configurations does not shows any trace of string connecting the quarks.

respectively. Notice that $\widetilde{L}_{c}<\widetilde{L}_{c}^{\prime}$.

The exact results are very close to the above approximate values. There exists a critical distance $L_{c}$ such that the Coulomb phase becomes unstable for quark-antiquark pairs with separations larger than $2 L_{c}$. At larger distances there is a symmetric unstable mode and for $L>L_{c}^{\prime}$ also an antisymmetric one. The critical size $L_{c}$ can reach arbitrary large values as $\alpha \rightarrow \sqrt{2}$ (see Figure 2) in agreement with the absence of unstable modes for $\alpha<\sqrt{2}$.

The profiles of those unstable solutions are displayed in Figure 3 and Figure 4. The symmetric mode, which is the most unstable mode (see Figure 3), exhibits a prominent thick string connecting the two quarks which points out to a picture of QCD where confinement would be driven by thick strings rather than by fundamental strings [21,22].

In summary, the behaviour of these negative modes implies that for intermediate values of the coupling constant the Coulomb phase is only stable on a heavy meson background for small sizes $L<L_{c}$. Whereas, for larger sizes $\left(L>L_{c}\right)$ the presence of negative modes leads to inconsistency of the background field expansion around Coulomb potentials.

Whether or not the instability means that at larger distances the theory is confining is unclear and remains as an open problem. Usually, the unstable modes get stabilized by higher order fluctuation selfinteractions. In that case the non-perturbative contribution of these modes would give rise to a linearly growing effective potential which would provide an evidence for confinement. But in this case the peculiar form of the unstable modes (2.3) implies that

$$
\left[\tau_{\mu}, \tau_{v}\right]=0
$$

which means that the quadratic approximation is exact, and stability cannot be restored by higher order terms in the usual way. 


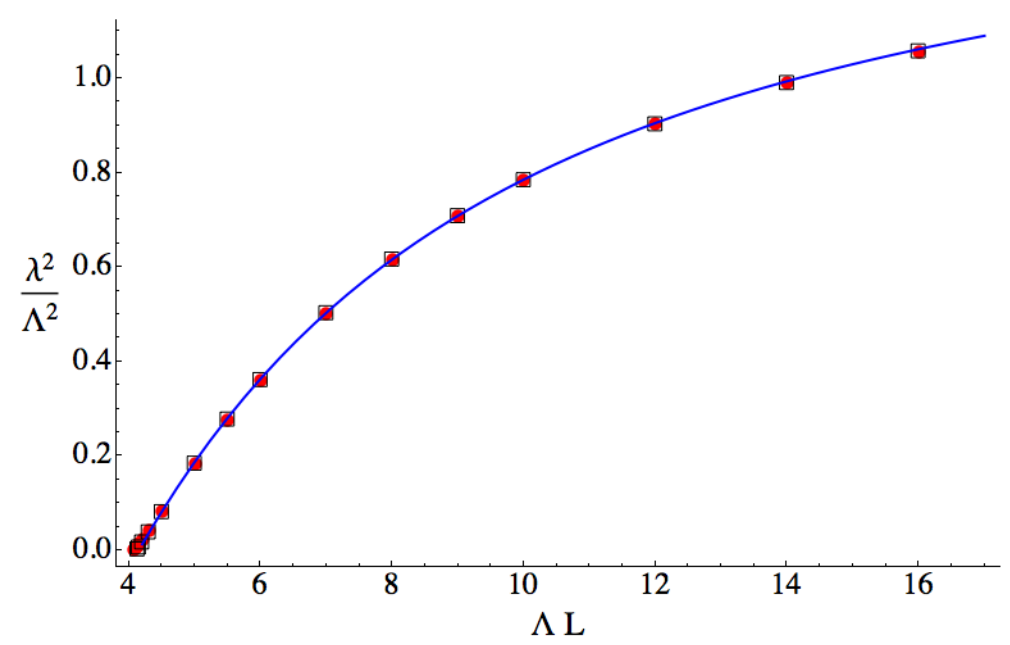

Figure 5: Behaviour of one of the infinite negative unstable modes of gluon fluctuations $-\lambda^{2}$ with heavy meson size $2 L$ at $\alpha=1.6$ in the strong coupling regime. Red dots are exact values for the symmetric mode whereas the squares are the eigenvalues of antisymmetric unstable modes. The blue curve represents the approximate values obtained by the analytic formula (3.1). Even if the mode becomes stable below a critical size, there are other unstable modes, not displayed on the picture, with larger negative values

\section{Conclusions}

In the strong coupling regime $\alpha>\frac{3}{2}$ there is an infinity of negative modes and even if each one becomes positive for close enough quark-antiquark pairs, there are always other modes that still remain negative for any distance between the quarks. In fact, in this case conformal symmetry is only partially broken, because of the periodicity of the boundary conditions (2.9) under the change $\Lambda \rightarrow \Lambda e^{\pi i / v}$, the theory remains covariant under this discrete group of scale transformations $\mathbb{Z}$. By the same reason the structure of the unstable modes follows the same periodic pattern as in the Efimov effect $\lambda_{n+1}^{2}\left(\mathrm{e}^{-i \pi / v} L\right)=\lambda_{n}^{2}(L) e^{2 \pi i / v}$ [8]. Thus, the Coulomb phase is always unstable in this regime for any size of the meson, which is compatible with the fact that the theory is confining in the strong coupling expansion.

The instabilities dues to negative modes of gluon fluctuations on a heavy meson background just mean that the expansion around a Coulomb background is not consistent for intermediate values of the coupling constant at large distances. In fact, these backgrounds are saddle points of the Euclidean functional integral and one can try to get a damping integrand just by changing the integration contour. However, the expansion is pathological for any choice of the integration contour, reflecting the fact that Coulomb phase does not hold at large distances for intermediate values of the coupling constant.

However, the functional integral is well defined. The pathology only means that the Coulomb saddle point is not relevant. The real physical problem is that the unstable modes do not point out to gauge field backgrounds where to find relevant saddle point configurations.

The results are surprising and support the Gribov picture of confinement. Even the critical value of the strong coupling constant $\alpha_{c}=\sqrt{2}$ on the presence of heavy quarks is of the same order of magnitude as the Gribov's critical value $\alpha_{G}$ for light quarks. The critical value $\alpha_{c}$ is slightly beyond the perturbative regime. This is due to the helicity one character of gluon fluctuations. 
If we consider light quarks in the same background the stability requirements provide a smaller critical value $\alpha_{F}=\sqrt{3} / 2$, closer to the Gribov critical value and inside the perturbative regime.

The transition from the asymptotic regime to a presumible confining regime for intermediate values of the strong coupling constant is a strong indication that QCD smoothly interpolates from an asymptotic freedom regime to a confinement regime.

The mere existence of a finite region of couplings $\alpha \in\left[\sqrt{2}, \frac{3}{2}\right]$, where the transition occurs for a given value of the $\alpha$ coupling, just by changing the separation between the two quarks, implies that the transition between the two regimes does not involves a critical phase transition. There is not a sharp separation at a given $\alpha$ coupling between two different regimes. There is a simple crossover. This is the first indication derived from first principles that QCD does not undergo a phase transition at intermediate energy scales. It will be interesting to analyse the effect of a finite temperature on QCD on the light of the new picture.

Acknowledgements: We thank J.C. Cuchí and J. M. Muñoz-Castañeda for collaboration in early stages of this work. We have been supported by the Spanish DGIID-DGA grant 2013-E24/2, the Spanish MICINN grants FPA2009-09638, FPA2012-35453 and CPAN-CSD2007-00042. A. S. thanks DGIID-DGA for a Ph. D. grant.

\section{References}

[1] V.N. Gribov, Talk at Perturbative QCD Workshop, Lund, Sweden, May 21Đ24, 1991. Preprint LU-TP- 91-7 (unpublished).

[2] V.N. Gribov, Orsay lectures on confinement LPTHE-ORSAY 92-60 (1992) [Arxiv preprint: hep-ph/9403218]; LPTHE-ORSAY 94-20 (1994) [Arxiv preprint: hep-ph/9404332] and LPT-ORSAY 99-37 (1999) [Arxiv preprint: hep-ph/9905285].

[3] V.N. Gribov, Eur. Phys. J. C10 (1999)70.

[4] V.N. Gribov, Gauge Theories and Quark Confinement. Collection of works, Phasis Publishing House, Moscow (2002), 357.

[5] C. Ewerz, Eur. Phys. J. C13 (2000) 503 [arXiv:hep-ph/0001038].

[6] Yu. L. Dokshitzer and D.E. Kharzeev, Ann. Rev. Nucl. Part. Sci. 54, (2004) 487.

[7] M. Asorey and A. Santagata, PoS ConfinementX (2012) 057.

[8] M. Asorey and A. Santagata, Nuovo Cimento C 36 (2013) 03.

[9] J.E. Mandula, Phys. Rev. D 14 (1976) 3497; Phys. Lett. B 67 (1977) 175; Phys. Lett. B 69 (1977) 495.

[10] M. Magg, Phys. Lett. B 74 (1978) 246; Phys. Lett. B 77 (1978) 199; Phys. Lett. B 78 (1978) 481; Nucl. Phys. B 158 (1979) 154.

[11] J.E. Mandula and L. McLerran, Phys. Lett. B 73 (1978) 193.

[12] A.J.G. Hey and J.E. Mandula, Phys. Rev. D 19 (1979) 1856.

[13] P. Sikivie and N. Weiss, Phys. Rev. Lett. 40 (1978) 1411; Phys. Rev. D18 (1978) 3809; Phys. Rev. D 20 (1979) 487.

[14] P. Sikivie, Phys. Rev. D 20 (1979) 877. 
[15] K.E. Cahill, Phys. Rev. Lett. 41 (1978) 599.

[16] I.B. Khriplovich, Sov. Phys. JETP 47 (1978) 18, Zh. Eksp. Teor. Fiz. 74 (1978) 37.

[17] R.A. Freedman, L. Wilets, S.D. Ellis and E.M. Henley, Phys. Rev. D 22 (1980) 3128.

[18] C.H. Oh, R. Teh and W.K. Koo, Phys. Lett. B 101 (1981) 337.

[19] L.J. Carson, R. Goldflam and L. Wilets, Phys. Rev. D 28 (1983) 385.

[20] L.J. Carson, Phys. Rev. D 29 (1984) 2355.

[21] J. Polchinski and L. Susskind, String theory and the size of hadrons [arXiv: hep-th/0112204].

[22] V. Vyas, Intrinsic Thickness of QCD Flux-Tubes [arXiv: 1004.2679]. 